\title{
Cisticercosis hepática: a propósito de un caso y revisión de la literatura
}

\author{
Guillermo Antonio Jiménez* \\ Juan Carlos Sabogal Olarte** \\ Daniel Upegui Jiménez $z^{* * *}$
}

\begin{abstract}
* Médico Patólogo. Compensar EPS. Docente temporal Universidad del Rosario. Bogotá. Colombia
**Médico cirujano, especialista en cirugía de hígado y páncreas. Hospital Universitario, Méderi. Bogotá. Colombia.

***Médico radiólogo, subespecialista en Abdomen y Pelvis. Hospital Universitario, Méderi. Bogotá. Colombia

Correspondencia: Dr. Guillermo Antonio Jiménez. Dirección: Cra 24 \#63C-69, Casa biociencias. Bogotá, Cundinamarca, Colombia. Correo electrónico: guillermoa.jimenez@urosario.edu.co
\end{abstract}

Resumen

La cisticercosis hepática es una enfermedad parasitaria bastante rara que consiste en el compromiso hepático por el cisticerco, con pocos casos reportados en la literatura, ninguno antes reportado en Colombia. Esta infección es producida por Cysticercus cellulosae, la forma larvaria del helminto Taenia solium. Es adquirida al consumir los huevos de la Tenia spp que se encuentran en la comida poco cocinada o el agua. En los casos reportados de cisticercosis hepática, el tratamiento se basa principalmente en el uso de antihelmínticos y en ocasiones quirúrgico. Se presenta el caso de un paciente mayor, indígena, quien consultó por melenas y dolor abdominal. En el examen físico se encontró hepatomegalia. Las imágenes diagnósticas plantean diagnóstico diferencial entre quiste hidatídico vs cistoadenoma/ cistoadenocarcinoma. El examen histopatológico sugiere compromiso por cisticercosis. Se reporta el caso de una cisticercosis hepática, con histología altamente sugestiva y hallazgos imagenológicos bastante inusuales a los previamente descritos en literatura. MÉD.UIS. 2020;33(2):101-108.

Palabras clave: Cisticercosis. Enfermedades parasitarias hepáticas. Enfermedades parasitarias.

Hepatic cysticercosis: a case report and review of the literature

Abstract

Hepatic cysticercosis is a quite rare parasitic disease consisting of hepatic involvement by the cysticercus, with few cases reported in the literature, none previously reported in Colombia. This infection is caused by Cysticercus cellulosae, the larval form of the Taenia solium helminth. It is acquired by consuming the Tenia spp eggs found in undercooked food or water. In reported cases of hepatic cysticercosis, treatment is based primarily on the use of anthelmintics and occasionally surgery. We present the case of an older, indigenous patient who consulted for melena and abdominal pain. Hepatomegaly is found on physical examination. Diagnostic images suggested a differential diagnosis between hydatid cyst vs. cystadenoma / cystadenocarcinoma. Histopathological examination suggested compromise due to cysticercosis. The case of hepatic cysticercosis is reported, with highly suggestive histology and imaging findings quite unusual to those previously described in the literature. MÉD.UIS. 2020;33(2):101-108.

Keywords: Cysticercosis. Liver diseases, parasitic. Parasitic Diseases.

¿Cómo citar este artículo?: Jiménez GA, Sabogal JC, Upegui D. Cisticercosis hepática: A propósito de un caso y revisión de la literatura. MÉD.UIS. 2020;33(2):101-108. doi: 10.18273/revmed.v33n22020012 


\section{Introducción}

La cisticercosis es una zoonosis de gran importancia en salud pública. Aproximadamente 50 millones de personas en el mundo entero se estiman tienen infección por cisticercosis, aunque probablemente sea mayor, ya que un elevado número de las infecciones son subclínicas'. Datos epidemiológicos a nivel mundial indican que la prevalencia varía porcentualmente en un mismo país y entre diferentes países, estando directamente relacionada con malas condiciones socioeconómicas, falta de saneamiento y de agua potable en zonas donde los cerdos se alimentan al aire libre y tienen fácil acceso a las heces humanas². Esta zoonosis es endémica en países de Latinoamérica, la mayor parte del África Subsahariana y grandes regiones de Asia ${ }^{3}$. En un estudio del 2013 realizado en Colombia, mostró que la seroprevalencia general es del $8,5 \%$, con un rango variable entre el $0,53 \%$ al $40,19 \%$, siendo la más alta en el departamento del Vaupés y la más baja en el departamento de Caldas ${ }^{4}$.

La cisticercosis es causada por Cysticercus cellulosae, el estado larvario del helminto Taenia solium. Las larvas de T. solium son quistes con vesículas llenas de líquido las cuales contienen la estructura de fijación del gusano en su interior (denominado escólex). T. solium tiene ciclo de vida de dos hospedadores, el cual ocurre entre seres humanos y cerdos. Tanto los cerdos como los seres humanos pueden ser hospedadores intermediarios para la forma larvaria, mientras que los humanos son el único hospedador definitivo para la forma adulta de la larva. Normalmente, los quistes son ingeridos por el ser humano a partir de carne de cerdo contaminada, posteriormente el escólex evagina, se ancla a la pared intestinal y madura a la forma adulta (gusano en forma de cinta de 2 a 4 metros). Las proglótides (cada uno de los segmentos morfológicos en los cuales se divide el gusano) en estado grávido y los huevos fértiles microscópicos entran en contacto con el ambiente externo por medio de las heces. En zonas con mala disposición de excretas y cerdos no estabulados, estos pueden ingerir heces humanas contaminadas con los huevos de T. solium. Después de ingerirlos, los embriones son liberados de los huevos, cruzan el intestino llegando a la circulación y de allí a los tejidos periféricos, incluyendo el sistema nervioso central. El ciclo se completa cuando el ser humano consume carne de cerdo infectada. Igual que los cerdos, los humanos pueden desarrollar cisticercosis luego de consumir las proglótides y los huevos fértiles, por lo cual la mayoría de las infecciones ocurren por vía fecal-oral³.
El cisticerco compromete con mayor frecuencia el sistema nervioso central, seguido del tejido celular subcutáneo, los tejidos blandos y los ojos. Aunque puede afectar cualquier órgano ${ }^{5}$, el compromiso hepático es extremadamente raro. Vianna et al, en un estudio realizado en 1991, estudiaron la frecuencia de cisticercosis en 1520 protocolos de necropsia, de los cuales en 25 casos $(1,6 \%)$ se encontró cisticercosis. De estos casos, 24 (96\%) con neurocisticercosis, 2 (8\%) compromiso en corazón y músculo esquelético y en 1 (4\%) en hígado ${ }^{6}$. En este órgano, el compromiso por cisticercosis se evidencia por la formación de quistes o nódulos. Cuando la lesión es solitaria, usualmente permanece asintomática; por el contrario, cuando son lesiones múltiples, los signos y síntomas simulan hepatitis o un proceso neoplásico?.

El objetivo del artículo es reportar un caso de cisticercosis hepática, además de hacer una revisión de la literatura con respecto a la clínica, hallazgos radiológicos, histológicos y tratamiento.

\section{Reporte de caso}

Hombre de 65 años, procedente de la ciudad de Mitú, capital de Vaupés, Colombia, de etnia indígena. El paciente es traído por una Organización No Gubernamental al Hospital Universitario MayorMéderi por presentar un cuadro clínico de dos días de evolución consistente de dolor abdominal inespecífico acompañado de deposiciones melénicas y emesis. Paciente describe sensación de masa en hipocondrio derecho de ocho meses de evolución.

Al examen clínico se encontró paciente hemodinámicamente estable, frecuencia cardíaca de 89 latidos por minuto, frecuencia respiratoria de 18 respiraciones por minuto, presión arterial de 95/72 mmHg, con saturación del $95 \%$ al aire ambiente. En el examen físico se encuentra un abdomen blando con hepatomegalia marcada y tacto rectal positivo para melenas.

En los estudios clínicos iniciales (Ver Tabla 1), donde se evidencia leucocitosis severa por lo cual se sospecha un síndrome mieloproliferativo vs reacción leucemoide. Por la hepatomegalia hallada en el examen físico se le ordena una ecografía abdominal. En este estudio se observó una masa hepática. El informe sugiere que debe ser caracterizada por estudios escanográficos con medio de contraste. 
Otros estudios solicitados fueron los siguientes: VIH 1 y 2, antígeno S para hepatitis B y anticuerpos anti-hepatitis C, los cuales fueron todos negativos; antígeno carcinoembrionario: $3,92 \mathrm{U} / \mathrm{ml}$; Alfafetoproteína: 4,73 U/ml; LDH: $701 \mathrm{U} / \mathrm{L}$; Endoscopia de vías digestivas altas: Úlcera gástrica Forrest III sin sangrado activo. La tomografía de abdomen y pelvis describen una lesión multiquística en segmentos V, VI y VII del hígado con ruptura al árbol biliar, sin poderse descartar infección. Por los hallazgos descritos, señalan como primera posibilidad quiste hidatídico con diagnósticos diferenciales entre cistoadenoma vs cistoadenocarcinoma de vía biliar intrahepática vs absceso hepático (Ver Figuras $1 \mathrm{~A}$ Y 1B). Por las anormalidades descritas en las imágenes, se decidió interconsultar el caso a cirugía hepatobiliar el cual solicita resonancia magnética abdominal para poder hacer una mejor caracterización de la lesión. Se inició cubrimiento antibiótico con ampicilina-sulbactam más metronidazol por sospecha de absceso hepático. Por los hallazgos en el hemograma, se interconsulta el caso a hemato-oncología, el cual sospechó un síndrome mieloproliferativo crónico, por lo cual se decidió solicitar aspirado más biopsia de médula ósea y JAK2 ambulatorio.

Tabla 1. Estudios de laboratorio solicitados al ingreso

\begin{tabular}{|l|c|}
\hline Estudio & Resultado \\
\hline Leucocitos & $69.30 \mathrm{~mm} 3$ \\
\hline Hemoglobina & $14.4 \mathrm{~g} / \mathrm{dL}$ \\
\hline Hematocrito & $47,20 \%$ \\
\hline Plaquetas & $312.000 \mathrm{~mm} 3$ \\
\hline Neutrófilos & $67,00 \%$ \\
\hline Nitrógeno uréico & $14.8 \mathrm{mg} / \mathrm{dL}$ \\
\hline Bilirrubina total & $0.29 \mathrm{mg} / \mathrm{dL}$ \\
\hline Bilirrubina directa & $0.13 \mathrm{mg} / \mathrm{dL}$ \\
\hline Aspartato aminotransferasa & $32 \mathrm{mg} / \mathrm{dL}$ \\
\hline Alanino aminotransferasa & $34 \mathrm{mg} / \mathrm{dL}$ \\
\hline Fostasa alcalina & $311 \mathrm{mg} / \mathrm{dL}$ \\
\hline Sodio & $136 \mathrm{mEq} / \mathrm{l}$ \\
\hline Potasio & $5.0 \mathrm{mEq} / \mathrm{l}$ \\
\hline Cloro & $98 \mathrm{mEq} / \mathrm{l}$ \\
\hline Calcio total & $9.6 \mathrm{mg} / \mathrm{dL}$ \\
\hline Deshidrogenasa láctica & $701 \mathrm{Jl} / \mathrm{dL}$ \\
\hline Proteína C reactiva & \\
\hline
\end{tabular}

Fuente: autores.

Al paciente se le realiza la resonancia magnética solicitada por cirugía hepatobiliar, en la cual se describe una masa quística, multiloculada de paredes y septos gruesos, la cual realza con medio de contraste, hipointensa en fase hepatocito específico, con diagnósticos diferenciales entre un cistoadenoma biliar vs absceso hepático (Ver Figura 2).
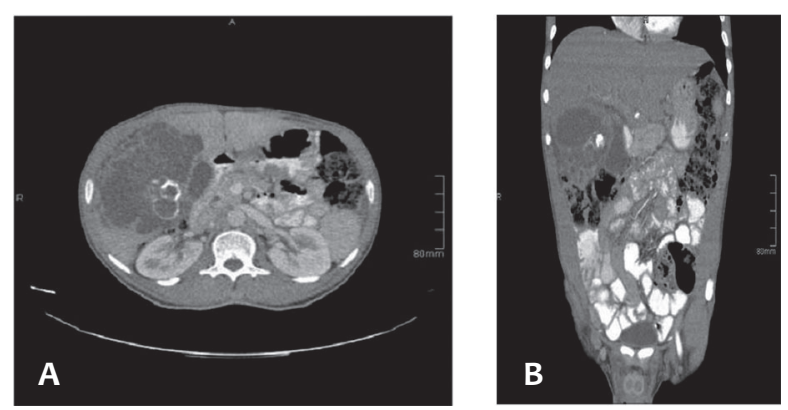

Figura $1 \mathrm{~A}$ y 1B. Tomografía axial computarizada con medio de contraste oral y endovenoso. plano axial (fig1a) y coronal (fig1b). En el lóbulo hepático derecho se observa una lesión quística multiloculada de paredes y septos gruesos con calcificaciones dismórficas.

Fuente: autores.

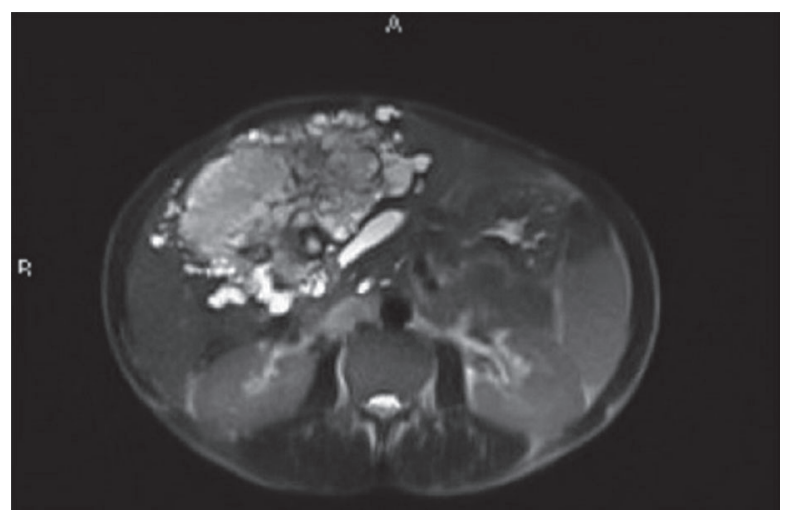

Figura 2. Resonancia magnética en plano axial potenciada en T2. Lesión quística multiloculada de paredes y septos gruesos con contenido heterogéneo de predominio hiperintenso.

Fuente: autores.

Se revisaron nuevamente las imágenes y la historia clínica en junta multidisciplinaria y se concluye que el cuadro clínico podría corresponder a un absceso hepático versus cistoadenocarcinoma, por lo cual se consideró manejo quirúrgico.

En el día 19 de hospitalización fue llevado a cirugía. Intraoperatoriamente, se evidenció un hígado de apariencia cirrótica con cambios de hígado graso. En el lóbulo hepático derecho presencia de una gran masa con extensión a los segmentos IVB y $\vee$ junto con hipertrofia significativa del lóbulo hepático izquierdo. Se realiza hepatectomía derecha extendida enviándose dicha muestra a patología.

Paciente ingresa a unidad de cuidados intensivos. En el posoperatorio inmediato en condiciones regulares 
con tendencia a la hipotensión, taquicárdico, deshidratado, gases arteriales ( $\mathrm{pH}: 7,19 ; \mathrm{PaCO}_{2}: 21,6$ mmHg; $\mathrm{PaO}_{2}: 76,5 \mathrm{mmHg} ; \mathrm{HCO}_{3}$ :: 8,2 mmHg; Lactato: $14 \mathrm{mg} / \mathrm{dL}$; PAFI $273 \mathrm{mmHg}$ ) y otros paraclínicos postoperatorios (conteo de leucocitos: 144,23 mm; Neutrófilos 92,4\%; hemoglobina $9 \mathrm{~g} / \mathrm{dl}$; plaquetas: $175.000 \mathrm{~mm}^{3}$; bilirrubina total $1,14 \mathrm{mg} / \mathrm{dL}$; bilirrubina directa: 0,93 mg/dL; BUN: 28,7 mg/dL; PT: 29,5; PTT: 32,9; INR 2,23). Por los hallazgos anteriores más el estado clínico del paciente, se concluyó que dicho cuadro corresponde a falla hepática y síndrome hepatorrenal. Se recibió al mismo tiempo el resultado de la citometría de flujo el cual indica no sugestivo de neoplasia hematolinfoide.

Al segundo día posoperatorio (día 21 de hospitalización) presentó deterioro cardiovascular, choque refractario con noradrenalina y vasopresina, progresando a bradicardia extrema y posterior asistolia, falleciendo finalmente el paciente. No se realizó necropsia.

En la evaluación histológica, se observa hígado con extensas zonas fibróticas, con infiltrado inflamatorio crónico. Se evidencia formación de cavidades con presencia de estructura parasitaria, tapizada por una cutícula externa eosinófila compuesta por células de núcleo pequeño. Estroma mixoide. El escólex del cisticerco no logró ser identificado (Ver Figuras 3A y B). Por las características morfológicas, los hallazgos son altamente sospechosos de compromiso por cisticercosis.

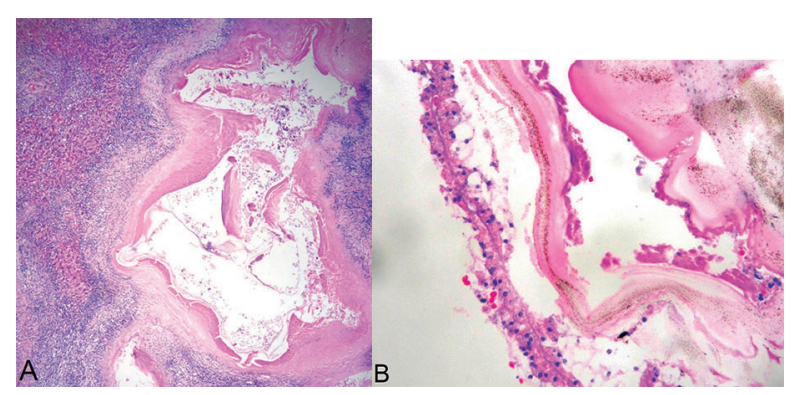

Figuras 3A y 3B. Al bajo aumento se observa hígado con infiltrado inflamatorio crónico intenso, el cual altera la arquitectura hepática. Formación de cavidades quísticas con tejido fibrótico alrededor. Al alto aumento se observa fragmentos del parásito, con cutícula eosinófila y parte de las células parasitarias.

Fuente: autores.

\section{Discusión}

La cisticercosis hepática es un desorden raro, de los cuales hay pocos casos reportados en la literatura ${ }^{6,8-14}$. Una síntesis de algunos de los casos reportados puede observarse en la Tabla 2. En los casos descritos en la literatura, casi todos los pacientes son hombres en un amplio rango de edad (entre 14 a 69 años). Las manifestaciones clínicas varían dependiendo de la localización y el número de las lesiones. En la aproximación diagnóstica se usó como primera herramienta, en la mayoría de los casos, la ecografía seguida de la tomografía axial y de la resonancia magnética nuclear. Para el tratamiento, lo más común fue utilizar la combinación de esteroides más un antiparasitario (praziquantel o albendazol).

La ecografía de alta resolución es la técnica inicial y de mejor rendimiento para evaluar la cisticercosis en tejidos blandos como en hígado. Otras técnicas de imagen tales como la tomografía computarizada y la resonancia magnética también pueden ser útiles. En la evaluación radiológica se evidencian quistes en varios estadios de evolución (vivo, muerto, en degeneración y calcificado) y por ello se observan diferentes patrones imagenológicos. Los quistes vivos típicamente se visualizan con fluido en su interior, bien circunscrito, redondo, con un nódulo mural excéntrico (el cual corresponde al escólex invaginado). Si la larva está viva, el fluido del quiste es claro. Si por el contrario, la larva está muerta, existe edema alrededor del quiste con realce de la pared, ambas a causa de la respuesta inflamatoria del hospedero. La inflamación crónica puede mimetizar un pseudotumor y al degradarse la larva, se genera una inflamación granulomatosa. El escólex no es siempre visualizado a causa del colapso parcial de los quistes con salida de este luego de la ruptura. Los cambios inflamatorios son observados de mejor manera en $\mathrm{T} 2$ con supresión de grasa y en imagen de resonancia magnética contrastada ${ }^{13}$. Este caso presentó la dificultad de no evidenciar las imágenes radiológicas características para la confirmación de infección por cisticercos, se observaron por imágenes, lesiones multiloculadas y septadas, siendo un hallazgo imagenológico bastante inusual. Pues en los reportes publicados de cisticercosis hepática se describen radiológicamente como lesiones quísticas uniloculadas. 
Tabla 2. Síntesis de algunos de los casos reportados.

\begin{tabular}{|c|c|c|c|c|c|c|c|}
\hline Autores & Año & Edad & Sexo & Antecedentes & Clínica & Aproximación diagnóstica & Tratamiento \\
\hline$V_{i a n n^{a 6}}$ & 1991 & ND & ND & ND & ND & $\begin{array}{l}\text { Diagnóstico realizado en necropsia, } \\
\text { encontrando compromiso } \\
\text { esquelético más compromiso } \\
\text { hepático. }\end{array}$ & ND \\
\hline Sickel $^{8}$ & 1995 & 62 & M & $\begin{array}{l}\text { Enfermedad } \\
\text { coronaria. } \\
\text { Adenocarcinoma } \\
\text { colónico. }\end{array}$ & $\begin{array}{l}4 \text { semanas de fiebre nocturna } \\
\text { y pérdida de } 10 \text { kilos de } \\
\text { peso. Al examen físico, } \\
\text { hepatomegalia. }\end{array}$ & $\begin{array}{l}\text { Ultrasonido seguido de TACC } \\
\text { que mostró alteración de } \\
\text { la ecogenicidad. Se realizó } \\
\text { laparatomía exploratoria, donde } \\
\text { se toma biopsia y se confirma el } \\
\text { diagnóstico }\end{array}$ & $\begin{array}{l}\text { Prednisolona y } \\
\text { praziquantel. }\end{array}$ \\
\hline Vijayaraghavan $^{9}$ & 2003 & 45 & M & ND & Síntomas de dispepsia & $\begin{array}{l}\text { Ecografía que mostró numerosas } \\
\text { calcificaciones pequeñas elípticas } \\
\text { vistas en hígado, mesenterio y } \\
\text { grasa retroperitoneal. Una lesión } \\
\text { en el pecho se realizó la excisión } \\
\text { confirmando cisticercosis por } \\
\text { histopatología }\end{array}$ & ND \\
\hline Sathyanarayanan ${ }^{10}$ & 2011 & 25 & M & ND & $\begin{array}{l}2 \text { meses de dolor de cabeza } \\
\text { y vómito. Al examen físico } \\
\text { presencia de hepatomegalia y } \\
\text { rigidez de nuca. }\end{array}$ & $\begin{array}{l}\text { Se realizó ultrasonido que mostró } \\
\text { presencia de múltiples quistes con } \\
\text { escolex en su interior. Se confirmó } \\
\text { el diagnóstico con ELISA para } \\
\text { detectar IgG para cisticercosis }\end{array}$ & Albendazol \\
\hline Singh $^{11}$ & 2012 & 16 & M & $\begin{array}{l}\text { Trabajador rural y } \\
\text { vegetariano }\end{array}$ & $\begin{array}{l}\text { Convulsiones tónico clínicas } \\
\text { generalizadas por } 1 \text { año, } \\
\text { dolor de cabeza y vómito } \\
\text { por } 3 \text { meses. Hace } 2 \text { meses } \\
\text { aparición de nódulos en piel. }\end{array}$ & $\begin{array}{l}\text { RMN mostró lesiones quísticas, } \\
\text { hiperintensas en T2 e hipointensa } \\
\text { en T1 en cerebro y músculo. TAC } \\
\text { halló lesiones quísticas en hígado } \\
\text { y bazo. }\end{array}$ & $\begin{array}{l}\text { Prednisolona, } \\
\text { albendazol, más } \\
\text { antiepilépticos }\end{array}$ \\
\hline $\operatorname{Sen}^{12}$ & 2013 & 14 & $M$ & ND & $\begin{array}{l}3 \text { semanas de fiebre } \\
\text { intermitente. Luego, aparición } \\
\text { de múltiples nódulos } \\
\text { dolorosos en la espalda, } \\
\text { tronco y muslos. Al examen } \\
\text { físico, hepatomegalia }\end{array}$ & $\begin{array}{l}\text { Se realizó TACC que mostró } \\
\text { múltiples lesiones hipodensas } \\
\text { en hígado y en varios grupos } \\
\text { musculares. Ultrasonido se } \\
\text { describió la presencia de } \\
\text { estructuras similares a escolex. } \\
\text { Se realiza biopsia de las lesiones } \\
\text { musculares confirmando el } \\
\text { diagnóstico. }\end{array}$ & $\begin{array}{l}\text { Esteroides y } \\
\text { albendazol. }\end{array}$ \\
\hline Chaudhary ${ }^{13}$ & 2014 & 28 & M & $\begin{array}{l}\text { Alcoholismo } \\
\text { crónico y fumador }\end{array}$ & $\begin{array}{l}2 \text { meses de dolor abdominal } \\
\text { en zona superior derecha } \\
\text { recurrente acomopañada de } \\
\text { fiebre e ictericia. }\end{array}$ & $\begin{array}{l}\text { Ultrasonido mostró hepatomegalia } \\
\text { leve y presencia de multiples } \\
\text { quistes, los cuales estaban en } \\
\text { varios estadios de evolución. } \\
\text { Algunos de los quistes con } \\
\text { presencia de escolex. }\end{array}$ & $\begin{array}{l}\text { Esteroides y } \\
\text { albendazol. }\end{array}$ \\
\hline Bennesch $^{14}$ & 2018 & 69 & $\mathrm{~F}$ & $\begin{array}{l}\text { Tuberculosis } \\
\text { pulmonar y } \\
\text { aspergilosis } \\
\text { pulmonar. } \\
\text { neumonías a } \\
\text { repetición }\end{array}$ & $\begin{array}{l}\text { Múltiples lesiones en piel } \\
\text { sobreelevadas, entre } 2 \text { a } 10 \\
\text { cm de diámetro con tiempo } \\
\text { de evolución desconocido. }\end{array}$ & $\begin{array}{l}\text { Ecografia, TAC y RMN presencia de } \\
\text { lesiones quísticas en tejido celular } \\
\text { subcutáneo, músculos e hígado. } \\
\text { Se realiza biopsia de quiste que } \\
\text { muestra restos de tubo digestivo de } \\
\text { un parásito. }\end{array}$ & $\begin{array}{l}\text { Albendazol y } \\
\text { meprednisona. }\end{array}$ \\
\hline
\end{tabular}

TAC tomografía axial computarizada, TACC tomografía axial computarizada con contraste, RMN Resonancia magnética, ND No disponible. Fuente: autores. 
En la evaluación microscópica, el parásito vivo tiene un escólex con cuatro ventosas y una doble fila de ganchos. La pared del quiste tiene una capa externa cuticular, una media pseudoepitelial y una capa interna reticular ${ }^{15}$. Desafortunadamente no todos los elementos pueden estar presentes a través de las diferentes fases de evolución de esta enfermedad y la fragmentación del tejido extraído, por lo cual la biopsia o la aspiración por aguja fina puede mostrar pedazos de ganchos, escólex y de la capa espiral del cisticerco ${ }^{10}$. En la evaluación microscópica de la lesión del paciente se observó la formación de cavidades conformadas por material fibrótico, con presencia de estructura parasitaria, la cual posee cutícula externa eosinófila con estroma mixoide. El escólex no logró ser identificado. Por estas características histológicas, corresponden muy probablemente a larva del cisticerco. A causa de la prematura muerte del paciente y no contar en nuestro laboratorio con pruebas moleculares, estas no se realizaron, por lo cual se plantea como limitante en este caso $^{16}$.

El tratamiento médico de los quistes sin edema se basa en el uso de albendazol o praziquantel, dos medicaciones anti-helmínticas. La escisión quirúrgica se reserva a la cisticercosis aislada y aquellas asociadas a abscesos. Se recomienda un seguimiento de dos veces por semana por un periodo de 4 a 6 semanas, para evaluar la respuesta al tratamiento ${ }^{13}$.

Los diagnósticos diferenciales más importantes se encuentran el quiste hidatídico, la coenurosis humana y el cistoadenoma biliar/cistoadenocarcinoma. A continuación, se habla de cada uno de estos diagnósticos diferenciales propuestos.

El quiste hidatídico es una enfermedad causada por la larva del gusano Echinoccocus, la cual es adquirida al ingerir las heces de perros y lobos que tengan en su intestino la forma adulta del gusano. La mayoría de los pacientes tienen una lesión quística simple, localizada en un solo órgano y afectando principalmente a hígado y pulmones, donde el compromiso hepático ocurre en el $60-75 \%$ de los casos, (siendo el lóbulo derecho más afectado que el izquierdo) ${ }^{17}$. En general, en estadios iniciales es asintomático y los síntomas dependen de su tamaño, posición, efecto de masa y complicaciones de la ruptura o sobreinfección. Los síntomas más comúnmente descritos incluyen dolor en abdomen superior, sensación de masa, falta de apetito e ictericia y en casos de ruptura, colangitis, reacción alérgica y anafilaxis ${ }^{18-19}$. Imagenológicamente, el quiste hidatídico se diferencia de la cisticercosis, por la presencia en el primero de quistes con membranas flotantes (signo de lirio de agua) y la existencia de quistes hijas adyacentes ${ }^{20}$. Histopatológicamente, el quiste hidatídico se puede distinguir de la cisticercosis por la presencia de las 3 membranas parasíticas (externa, media e interna), y la presencia de succionadores, ganchos y corpúsculos calcáreos ${ }^{18}$.

En un caso reportado de quiste hidatídico hepático, Yuan et al., describe un paciente de 37 años, el cual consulta por dolor abdominal superior intermitente y fiebre. Se le realizan paraclínicos los cuales encuentran ALT de $50 \mathrm{U} / \mathrm{L}$. A causa de la sintomatología y los hallazgos de los paraclínicos, se le toman imágenes donde efectivamente encuentran una masa en lóbulo derecho, multiquística, con presencia de vesículas hijas. Paciente se le realiza resección quirúrgica de la lesión. En el estudio anatomopatológico, se encuentra una estructura parasitaria con membranas parasíticas. No se encontraron estructuras parasíticas adicionales. Se confirmó el diagnóstico de quiste hidatídico ${ }^{21}$. Aunque el cuadro clínico de este caso es muy similar al descrito en nuestro paciente, exceptuando la presencia de melenas y el desenlace fatal, se logran observar algunas diferencias, entre ellas, la descripción en el quiste hidatídico de vesículas hijas por radiología y en el estudio histológico la presencia de estructura parasitaria con membranas parasíticas características.

La coenurosis humana es una infección extremadamente rara, pues solo se han reportado 150 casos, esta es causada por el coenuri, el cual es el estadio larvario de la Taenia multiceps, T. serialis, T. brauni y T. glomerata. Los cánidos son los huéspedes definitivos, donde una variedad de mamíferos son los huéspedes intermedios. La infección humana se produce a partir de la ingestión accidental de huevos provenientes de comida o agua contaminada. La mayoría de los casos reportados ocurren como masas subcutáneas o intramusculares, y de forma más inusual llegan a afectar el sistema nervioso central. En este último se manifiesta clínicamente como dolor de cabeza severo, alteraciones de la personalidad, pérdida de peso, rigidez de nuca e hipertensión intracraneal. Por imágenes se puede realizar un diagnóstico presuntivo, siendo el estudio histopatológico esencial para el diagnóstico definitivo. Morfológicamente se puede distinguir de la cisticercosis por el número y características del escólex. Si este no puede ser identificado, la 
presencia de quistes invertidos es de gran ayuda ${ }^{22-23}$, los cuales no fueron observados en este caso.

En un caso reportado de coenurosis cerebral, Ali et al, se encontró que aunque histopatológicamente es similar a otros cestodos, con una capa cuticular externa y una capa reticular interna, en la coenurosis la capa cuticular externa es ondulante e invaginada ${ }^{24}$. En el caso de coenurosis, aunque histológicamente son muy similares, este caso reportado por Ali et al presentó quistes invertidos. Además, hay que tener en cuenta que nunca se ha reportado compromiso hepático por el coenuri.

En el cistoadenoma biliar y el cistoadenocarcinoma, se observa un realce de la pared con el contraste en la tomografía computarizada. Sin embargo, cuando existen calcificaciones en estos tumores, puede no observarse este realce ${ }^{25}$. Los tumores biliares son característicamente multiloculados, septados y con engrosamiento irregular de la pared ${ }^{26}$. Xinting et al describe 33 casos, de los cuales 19 son cistoadenomas y 14 fueron cistoadenocarcinomas. Los síntomas descritos en la mayoría los pacientes con cistoadenomas son distensión abdominal y dolor; En uno solo de los pacientes se describió náuseas, vómitos e ictericia. Imagenológicamente, se describen múltiples patrones, entre ellos, quistes multiloculares o uniloculares, con paredes irregulares engrosadas, con septos internos y proyecciones papilares. Los septos y paredes demuestran realce tras la administración de medio de contraste endovenoso. En los cistoadenocarcinomas, al igual que en los cistoadenomas, los pacientes consultan por distensión abdominal y dolor y puede acompañarse por fiebre. Las características radiológicas de los cistoadenocarcinomas fueron muy similares a la de los cistoadenoma ${ }^{27}$. Al comparar las imágenes radiológicas con nuestro caso, se observaron quistes multiloculares, con aumento del contraste intravenoso, por lo cual radiológicamente no fue posible diferenciarla de un cistoadenoma/cistoadenocarcinoma. En estos casos el estudio histológico es importante para poder hacer la diferenciación entre cistoadenoma / cistoadenocarcinoma y la cisticercosis hepática. En el cistoadenoma se observa la formación de quistes tapizados por epitelio columnar, cuboidal o plano de tipo biliar o mucinoso con un estroma de tipo ovárico. Si se observa atipia citológica e invasión corresponde a un cistoadenocarcinoma ${ }^{28}$. No se evidenciaron este tipo de hallazgos histológicos en el presente caso.

\section{Conclusiones}

El diagnóstico de cisticercosis hepática es un gran reto debido a su similitud tanto clínica como radiológica con otras entidades, tal como fue expuesto en este caso clínico, debido a su amplio espectro de presentación. Aunque la morfología en las imágenes radiológicas tiene un alto rendimiento diagnóstico, este puede no ser concluyente dependiendo del estadio de evolución de la lesiones. La biopsia es una herramienta diagnóstica esencial si no se tiene claridad frente a la etiología de las lesiones, ya sea para realizar estudio morfológico de la lesión como para realizar pruebas moleculares. En la mayoría de los casos reportados en la literatura, el tratamiento se basa en antihelmínticos. Aunque se presentó un desenlace fatal, en general es una entidad con buen pronóstico si es diagnosticada a tiempo. Adicionalmente, un diagnóstico oportuno permite que se puedan implementar medidas de salud pública en su comunidad para evitar futuros casos de infección por el cisticerco.

\section{Conflictos de interés}

Los autores declaran que no existen conflictos de interés.

\section{Consideraciones éticas}

El siguiente reporte de caso fue aprobado por el comité de ética médica de la Universidad del Rosario con visto bueno de la gerencia del servicio de patología. Según la resolución 008430 de 1993 dictada por el ministerio de Salud, en la cual se establecen las normas científicas, técnicas y administrativas para la investigación en salud, en el título II capítulo I, artículo 11. La presente investigación es clasificada dentro de la categoría: con riesgo mínimo. Paciente al ser hospitalizado firma un consentimiento informado el cual señala que: "4. Adicionalmente se me ha informado que en la institución por su carácter de Hospital Universitario participa personal en formación que coopera en la atención bajo la responsabilidad y supervisión directa del profesional docente. Así mismo se me ha explicado que los datos e imágenes de la historia clínica pueden ser utilizados con carácter académico, manteniendo siempre bajo confidencialidad mi identidad.". Se ha protegido completamente su identidad del paciente. 


\section{Referencias bibliográficas}

1. White AC. Cysticercosis: Epidemiology, transmission, and prevention [Internet]. UpToDate. 2018 [cited 1 February] Available from: https://www.uptodate.com/contents/ cysticercosis-epidemiology-transmission-and-prevention

2. Montresor A, Palmer K. Taeniasis/cysticercosis trend worldwide and rationale for control. Parasitol Int. 2006;55(Suppl):S301S303.

3. Garcia HH, Nash TE, Del Brutto OH. Clinical symptoms, diagnosis, and treatment of neurocysticercosis. Lancet Neurol. 2014;13(12):1202-15

4. Carolina A, Sánchez F, Pastrán SM, Vargas NS, Beltrán M, Enriquez $\mathrm{Y}$, et al. Cisticercosis en Colombia. Estudio de seroprevalencia 2008-2010. Acta Neurol Colomb. 2013;29(2):73-86.

5. Farrar J, Junghanss T, Lalloo D, Hotez PJ, Kang G, White NJ Manson's tropical diseases. 23 ${ }^{\mathrm{a}}$ ed. Londres: Elsevier; 2014

6. Vianna LG, Macedo V, Costa JM. Cisticercose músculo-cutânea e visceral--doença rara? [Musculocutaneous and visceral cysticercosis: a rare disease?]. Rev Inst Med Trop Sao Paulo. 1991;33(2):129-36.

7. Zimmermann A. Tumor-like Parasitic Lesions of the Hepatobiliary Tract: Echinococcosis and Cysticercosis. In: Tumors and TumorLike Lesions of the Hepatobiliary Tract Springer International Publishing; 2016. p. 1-15.

8. Sickel JZ, Fultz PJ, Penwarden B, Laczin J. Hepatic cysticercosis Report of an unusual case. J Clin Gastroenterol. 1995;20(2):160-3.

9. Vijayaraghavan SB. Sonographic appearances in cysticercosis. J Ultrasound Med. 2004;23(3):423-7.

10. Sathyanarayanan V, Sambhaji C, Saravu K, Razak A, Polnaya A, Rao SN. A rare case of hepatic cysticercosis. Asian Pac J Trop Biomed. 2011;1(1): S139-40.

11. Singh N, Singh DK, Parihar A, Singh R. Disseminated cysticercosis: rare manifestation of a common disease. BMJ Case Rep. 2012.

12. Sen K, Karmakar P, Bandyopadhyay A, Santra T, Sarkar M. Cysticercosis involving muscles and liver only: A yet unknown entity. Ann Trop Med Public Heal. 2013;6(3):350-2.

13. Chaudhary V, Bano S, Kumar P, Narula MK, Anand R. Hepatic cysticercosis: a rare entity. Abdom Imaging. 2014;39(6):1182-5.

14. Olivares LM, Bennesch A, Fischer J, Coringrato M, Maronna E. Cisticercosis cutánea y hepática diseminada en una paciente con infección por HTLV-1. Dermatología Argentina. 2018;24(2):10810.

15. Kradin RL. Diagnostic Pathology of Infectious Disease. $2^{\mathrm{a}}$ ed. Philadelphia: Elsevier; 2017.

16. Yamasaki H, Nakao M, Sako Y, Nakaya K, Ito A. Molecular identification of Taenia solium cysticercus genotype in the histopathological specimens. Southeast Asian J Trop Med Public Health. 2005;36(4):131-4.

17. Agudelo Higuita NI, Brunetti E, McCloskey C. Cystic Echinococcosis. J Clin Microbiol. 2016;54(3):518-23.

18. McManus DP, Gray DJ, Zhang W, Yang Y. Diagnosis, treatment, and management of echinococcosis. BMJ. 2012;344:E3866.

19. Wen H, Vuitton L, Tuxun T, Li J, Vuitton DA, Zhang W, et al. Echinococcosis: Advances in the 21st Century. Clin Microbiol Rev. 2019;32(2):E00075-18

20. Brunetti E, Tamarozzi F, Macpherson C, Filice C, Piontek MS Kabaalioglu A, et al. Ultrasound and Cystic Echinococcosis. Ultrasound Int Open. 2018;4(3): E70-E78.

21. Yuan WH, Lee RC, Chou YH, Chiang JH, Chen YK, Hsu HC. Hydatid cyst of the liver: a case report and literature review. Kaohsiung J Med Sci. 2005;21(9):418-23.

22. Lescano AG, Zunt J. Other cestodes: sparganosis, coenurosis and Taenia crassiceps cysticercosis. Handb Clin Neurol. 2013;114: 335-45.

23. Dailey Garnes NJM, Serpa JA, White AC. Taenia (Multiceps multiceps and Taenia serialis. In Principles and Practice of Pediatric Infectious Diseases. Elsevier; 2018. p. 1410-1.e1.

24. Ali SM, Reddy PS, Venugopal S, Chhabra M, Mahadevan A. Cerebral Coenurosis Masquerading as Malignancy: A Rare Case Report from India. J Neurosci Rural Pract. 2019;10(2):367-70.

25. Bächler P, Baladron MJ, Menias C, Beddings I, Loch R, Zalaquett E, et al. Multimodality Imaging of Liver Infections: Differential Diagnosis and Potential Pitfalls. Radiographics. 2016;36(4):1001-23.

26. Soares KC, Arnaoutakis DJ, Kamel I, Anders R, Adams RB, Baue TW et al. Cystic neoplasms of the liver: biliary cystadenoma and cystadenocarcinoma. J Am Coll Surg. 2014;218(1):119-28.

27. Sang X, Sun Y, Mao Y, Yang Z, Lu X, Yang H, et al. Hepatobiliary cystadenomas and cystadenocarcinomas: a report of 33 cases. Liver Int. 2011;31(9):1337-44.

28. Saxena R. Benign and malignant tumors of bile ducts. Practical hepatic pathology: A Diagnostic Approach. $2^{\circ}$ ed. Philadelphia: Elsevier/Saunders; 2018. p. 545-554. 